\title{
Log-transform Weighted Total Variation for Image Smoothing
}

\author{
Qiegen Liu \\ Department of Electronic \\ Information Engineering, \\ Nanchang University, \\ Nanchang, China
}

\author{
Li Zhu \\ Department of Electronic \\ Information Engineering, \\ Nanchang University, \\ Nanchang, China
}

\author{
Jianhua Wu \\ Department of Electronic \\ Information Engineering, \\ Nanchang University, \\ Nanchang, China
}

\begin{abstract}
Since the input image in computer vision and graphics containing various texture/structure patterns provides rich visual information, how to properly decompose them is a challenging problem. Recent developments in high-contrast detail smoothing show that how they define edges and how this prior information guides smoothing are two key points. In this paper, we present a novel Log-transform weighted total variation (LWTV) method, which employs the signed gradient summation of Log-transform pixels at neighbor window as data-fidelity weight. Specifically, LWTV substantially improves the decomposition for the regions with faint pixelboundary and alleviates the drawback of slightly blurry. Experimental results demonstrate that the proposed method has appearance performance on image with abundant uniform textural details.
\end{abstract}

\section{Keywords}

Image smoothing, structure preserving, texture eliminating, Log-transform, total variation.

\section{INTRODUCTION}

Many problems in computer vision and computer graphics involve estimating some spatially varying image-content from original noisy image. One important requirement of such estimation is feature-preserving filtering, which became a fundamental tool in many applications. Unfortunately, feature-preserving filtering is inherently challenging, because it is difficult to distinguish features from noise, especially where high-contrast trivial details needed to be smoothed. Structure-preserving smoothing with jointly textureeliminating greatly improves the image editing and rendering $[1,2]$. There are some existing feature-preserving filtering methods to deal with this problem, due to the Xu's pioneer work of extracting major structures from texture images. These approaches can be roughly classified into two categories, i.e. spatial filter $[2,3,4,5]$ and variational model $[1,6,7,8]$.

The first category of high-contrast detail smoothing methods exploits the spatial filtering directly. Bilateral filtering (BF) [3] is a widely used model for removing noise from images while simultaneously performing detail flattening and edge preservation. It averages the nearby pixels by calculating weights from spatial and range domain, and smoothes lowcontrast regions while preserving high-contrast edges. Recognizing that BF works poorly to smooth out highcontrast textures, Su et al. developed an edge-preserving texture suppression filter based on joint BF filtering scheme [4]. Inspired by the fact that extending the concept of neighborhood in a non-local way to potentially include more pixels such that may in favor of smoothing, the Regcovsmooth developed by Karacan et al. used the second order statistic descriptor region covariance as a similar weight to average the pixels in a squared neighborhood [5]. Regcovsmooth may smooth the edge when works on image containing number of cartoon patterns.

Variational models follow the energy minimization framework with a data term and a smooth term $[1,6,7,8]$. The data term measures the disagreement between the filtered signal $s$ and the original signal $f$, while the smooth term measures the extent to which the filtered signal is not piecewise smooth. i.e.

$$
s=\arg \min _{s}\{G(f, s)+\lambda J(s)\}
$$

where $\lambda$ is a non-negative parameter controlling the weight of the smooth term. The design of the data term $G(f, s)$ is usually straightforward. For instance, the squared L2 distance between the filtered signal and the original signal $\|f-s\|_{2}^{2}$ is often used. The choice of the smooth term $J(s)$ is a critical issue. A representative work is the total variation (TV) [6], which uses L1-norm based regularization constraints to penalize large gradient magnitudes. In its original formulation, TV model provides fairly good separations for structure from texture. Some studies extended the standard TV formulation with different norms for both regularization and data fidelity terms, and demonstrated that more robust norms could improve the performance of image decomposition $[1,7,8]$. In [7], Farbman et al. proposed a robust method with the weighted least square (WLS) measure. The L0smoothing presented by $\mathrm{Xu}$ et al. [8] used $l_{0}$ term instead of $l_{1}$ term to directly measure the gradient sparsity in the context of image smoothing. Unfortunately, like BF filter, WLS and L0smoothing also work poorly for tackling the image with non-uniform texture details, since they are merely based on image contrasts or gradients. Later, Xu et al. proposed method relative total variation (RTV) to smooth mosaic images with highly texture patterns [1]. The efficiency of RTV depends on the defined windowed total variation (WTV) and windowed inherent variation (WIV) involving spatial information. Despite of achieving impressive results, RTV may still mistakenly identify image structures as textures and meanwhile blur the edges.

In summary, the differences among these approaches lie in how they define edges and how this prior information is used to guide smoothing. How to design an appropriate 
weight/kernel feature to effectively distinguish high-contrast and fine-scale details from major structures is a critical task. In this paper, a novel Log-transform weighted total variation (LWTV) method is proposed, which employs Logtransform in the weight construction. Unlike the recent RTV proposed on the basis of statistical verification, our motivation is derived from the interpretation of weighted total variation and the impressive nonlinear property of Log-transform. Our idea aims to incorporate the Log-transform into an adaptive data-fidelity weight term. By integrating the local region Logtransform based gradient weight information into the smoothing process, LWTV satisfactorily distinguishes texture from the main structures. The rest of this paper is organized as follows. Section 2 presents The LWTV algorithm. Experimental results are presented in Section 3, and Section 4 concludes the paper.

\section{ALGORITHM LWTV}

In this section, after reviewing the applications of Logtransform in image processing and computer vision, we developed a Log-transform weighted total variation (LWTV) model for smoothing out high-contrast texture details. The superiority of the designed weight to that of RTV was explained in detail. Finally, an effective algorithm solver with iterative mechanism was provided.

\subsection{Review of Log-Transform in Image Processing}

Logarithmic transformation (Log-transform) is a basic and popularly used transformation in image processing community. The mathematical formulation of traditional Logtransform is $s=c \log (1+r), r \geq 0$, where $c$ is a constant. The transformation curve depicted in Fig. 1(a) maps a narrow range of low-level grey scale intensities into a wider range of output values and similarly maps the wide range of high-level grey scale intensities into a narrow range of high level output values, which prefers to compress the dynamic range of images with large variations in pixel values or Fourier spectrum image [9]. Usually, applying log-transform to an image will expand its low valued pixels to a higher level and have little effect on higher valued pixels, hence it enhances minor image detail (or contrast) with lower intensity values. In this paper, we use the mathematical form $s=\log (r), 1 \geq r \geq 0$ displayed in Fig. 1(b), where the image is normalized to $[0,1]$ in advance.

Log-transform has been used in diverse image tasks. In [10], Fattal et al. presented a gradient domain high dynamic range (HDR) compression, which applied gradient attenuation in the logarithm of the luminances. The well-known edge-ware filter WLS [7] uses the gradient of the logarithm value as a weighting term of the least squares problem. Obviously, the role of the weight in the regularization term is to force the filtered results at regions where gradient is large to be as close as possible to the input image, meanwhile that at other regions to be smoothed. As discussed in the introduction, since WLS only uses pixel intensity contrasts (the gradient of Logtransform of its intensity) as guidance to smooth image, it fails to suppress the high-contrast texture details. Recently, $\mathrm{Li}$ et al. [11] proposed Local Log-Euclidean covariance matrix for image representation. Their defined covariance matrices form a Lie group equipped with Euclidean space structure, which enables common Euclidean operations in the logarithm domain. Experiment results showed its promising performances in the applications of texture classification and object tracking.

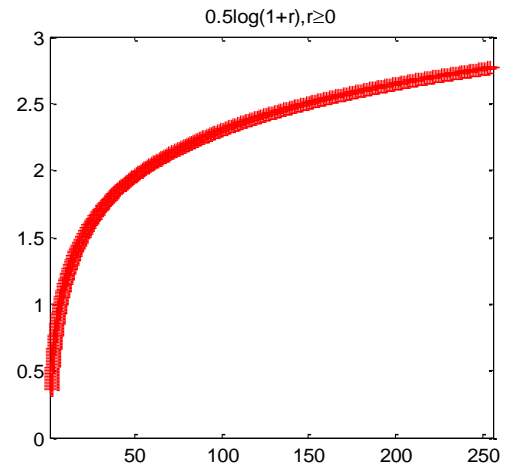

(a)

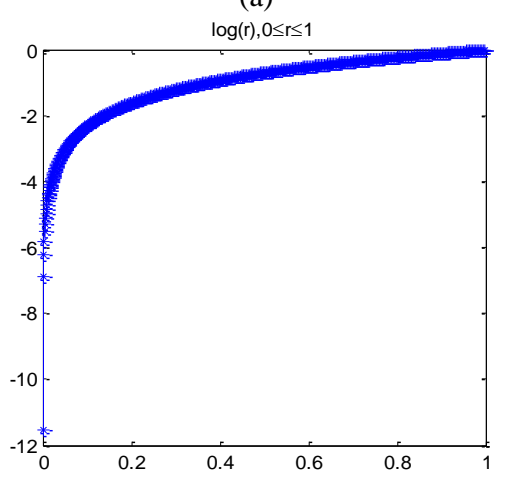

(b)

Fig 1: Illustration of Log-transform. $s=0.5 \log (1+r), r \geq 0$, (b) $s=\log (r), 1 \geq r \geq 0$.

(a)

\subsection{Algorithm Outline}

Recently, based on the statistical verification that the defined RTV measure of texture and main structure exhibit different properties that make them decomposable. Xu et al. presented an optimization framework, in which meaningful content and textural edges are penalized differently. i.e.

$$
s=\arg \min _{s}\left\{\|f-s\|_{2}^{2}+\lambda \sum_{i}\left[\frac{D_{x} s(i)}{L_{x} s(i)+\varepsilon}+\frac{D_{y} s(i)}{L_{y} s(i)+\varepsilon}\right]\right\}
$$

where $D_{x} s(i)=\sum_{j \in R(i)} g_{i, j}\left|\left(\partial_{x} s\right)_{i}\right| . L_{x} s(i)=\sum_{j \in R(i)}\left|g_{i, j}\left(\partial_{x} s\right)_{i}\right|$ are the weights. $g_{i, j}$ is a Gaussian weighting function with standard deviation $\sigma$ [1]. $i$ and $j$ are pixel indexes, $x$ and $y$ are pixel coordinates. In essence, its success lies on the guidance of weight $L$ in the regularization term. i.e, the resulting $L$ in a window that only contains texture is generally smaller than that in a window also including structural edges. An intuitive explanation is that a major edge in a local window contributes more similar-direction gradients than textures with complex patterns. Hence, the high-contrast details, whose smaller $L$ values implying more total variation penalty will be forced, will be smoothed more heavily. However, one major drawback of RTV is that it could mistake part of structures as texture, if they are visually similar in scales or interweave in the same region. One example is shown in Fig. 2, where two structures regions of RTV result in Fig. 2(c) are not well preserved. It is because that the scale and shape of these edges are overly close to those of the underlying texture. 
Motivated by the fact that the Log-transform may expand its valued pixels distribution and thus in favor of the separation of edges and textures, we propose a Log-transform weighted total variation (LWTV) model. Mathematically, by employing Log-transform in the weight total variation, the objective function can be written as:

$$
M_{s} \operatorname{in}\left\{\|f-s\|_{2}^{2}+\lambda \sum_{i}\left[L W_{x} s(i)\left|\left(\partial_{x} s\right)_{i}\right|+L W_{y} s(i)\left|\left(\partial_{y} s\right)_{i}\right|\right]\right\}
$$

where $L W_{x} s(i)=\left(\sum_{j \in R(i)}\left|g_{i, j}\left(\partial_{x} \log (s)\right)_{i}\right| / \sum_{j \in R(i)} g_{i, j}\right)^{-1}$ is the weight. The penalty in the $y$-directional dimension is the same as that in $x$-directional dimension.

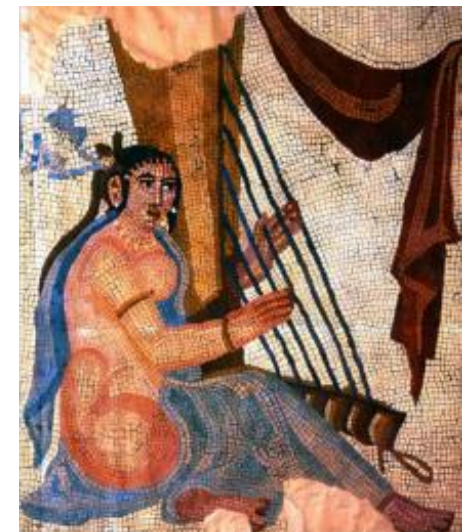

(a)

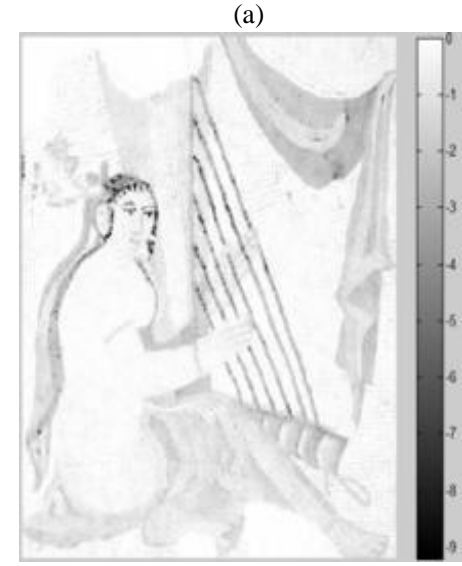

(d)
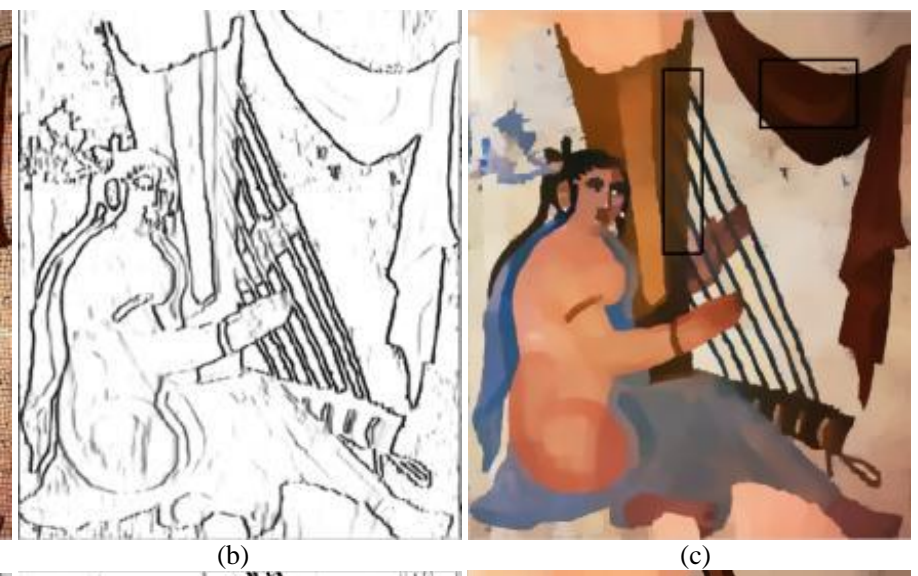

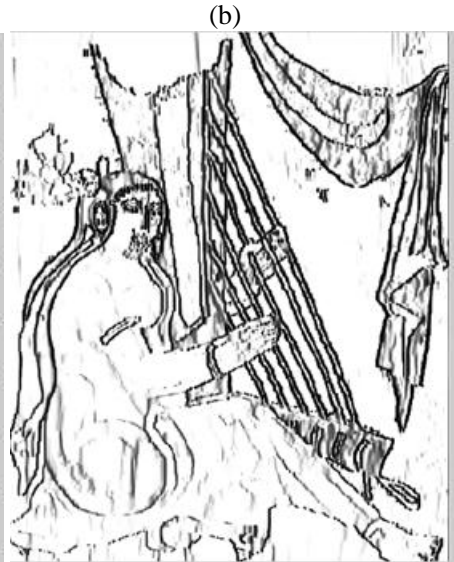

(e)

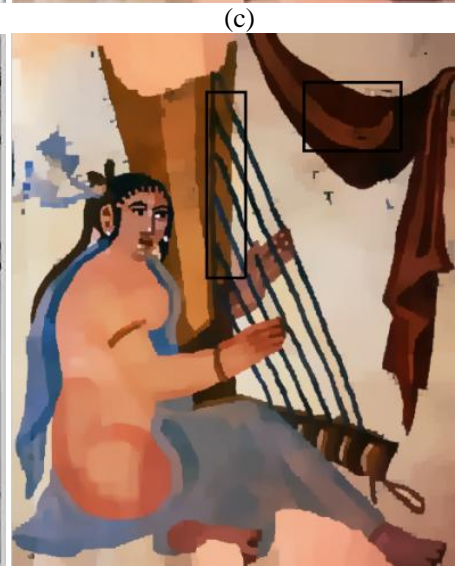

Fig 2: Demonstration of difference between RTV and LWTV. (a) The input image, (b) the inversion of normalized $L_{x}$, (c) RTV result, (d) Log-transform of (a), (e) $L W_{x}$ value, (f) LWTV result.

The advantage of the proposed model LWTV can be observed in the second line of Fig. 2. Firstly, the image depicted in Fig. 2(d) seems to be more distinct due to the Log-transform. Concretely, for the pixels cross the edge boundary, their difference will be more amplified by Log-transform than these pixels cross the texture regions. Consequently, the resulting $L W_{x}$ in Fig. 2(e) is more in accordance with our visual recognition of edge and texture. Finally, the LWTV result in Fig. 2(f) produces better separation effect.

\subsection{Algorithm Solver}

For the minimization problem Eq. (3), we adopt a relaxation reweighted strategy:

$$
L W_{x} s(i)\left|\left(\partial_{x} s\right)_{i}\right|+L W_{y} s(i)\left|\left(\partial_{y} s\right)_{i}\right|
$$

$$
\begin{aligned}
& \approx L W_{x} s(i) \frac{1}{\left|\left(\partial_{x} s\right)_{i}\right|+\varepsilon}\left(\partial_{x} s\right)_{i}^{2}+L W_{y} s(i) \frac{1}{\left|\left(\partial_{y} s\right)_{i}\right|+\varepsilon}\left(\partial_{y} s\right)_{i}^{2} \\
& =u_{x, i} w_{x, i}\left(\partial_{x} s\right)_{i}^{2}+u_{y, i} w_{y, i}\left(\partial_{y} s\right)_{i}^{2}
\end{aligned}
$$

where $u_{x, i}=L W_{x} s(i)$ and $w_{x, i}=1 /\left(\left|\left(\partial_{x} s\right)_{i}\right|+\varepsilon\right)$.

The second line in Eq. (4) is approximated by the Iteratively Reweighted Norm (IRN) approach proposed by Rodriguez et.al [12] . Eq. (3) can be written in a matrix form as follow:

$$
v_{s}=\arg \min _{v_{s}}\left\{\left(v_{f}-v_{s}\right)^{T}\left(v_{f}-v_{s}\right)+\lambda\left(v_{s}^{T} C_{x}^{T} U_{x} W_{x} C_{x} v_{s}+v_{s}^{T} C_{y}^{T} U_{y} W_{y} C_{y} v_{s}\right)\right.
$$

where $v_{s}$ and $v_{f}$ are the vector representation of $s$ and $f$ respectively. $C_{x}$ and $C_{y}$ are the Toeplitz matrices from the 
discrete gradient operators with forward difference. $U_{x}, W_{x}$, $U_{y}$ and $W_{y}$ are diagonal matrices whose elements are defined in Eq. (4). Similar to RTV, Eq. (5) is solved by a reweighted strategy, i.e. updates the block variables $U_{x}, W_{x}$, $U_{y}$ and $W_{y}$ as a function of $v_{s}$ calculated from the previous iteration and then updates $v_{s}$ by minimizing Eq. (5) with the last values of $U_{x}, W_{x}, U_{y}$ and $W_{y}$. Denoting $L^{k}=C_{x}^{T} U_{x}^{k} W_{x}^{k} C_{x}+C_{y}^{T} U_{y}^{k} W_{y}^{k} C_{y}$, thus the minimization of Eq. (5) is given by

$$
\left(1+\lambda L^{k}\right) v_{s}^{k+1}=v_{f}
$$

Since $\left(1+\lambda L^{k}\right)$ is a symmetric positive definite Laplacian matrix, efficient solvers are available for it. The whole optimization process is summarized as follows:

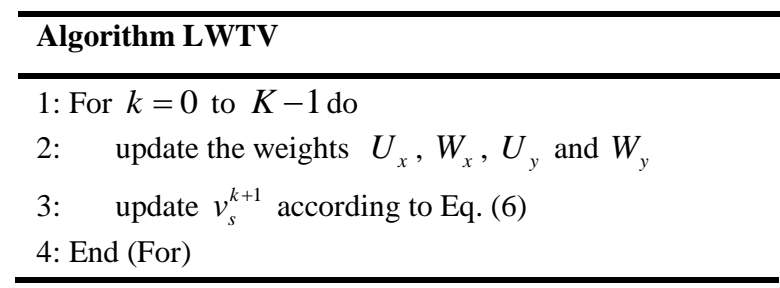

\section{EXPERIMENTAL RESULTS}

The performance of LWTV was evaluated on several experiments under different scenario, which aims to investigate the effect of imposing Log-transform signed gradient weight ${ }^{1}$. In the experiment, the proposed method was compared with some other techniques addressing highcontrast detail smoothing. The parameter settings are corresponding to each operator's own formulation and tuned with our best efforts for smoothing out high-contrast details while preserving major structures. All the test images are normalized to have a maximum magnitude of 1 .

Fig. 3 displays some decomposition examples with comparison to RTV, where the high-contrast details at the background have different shapes and patterns. Generally, RTV method usually produces false results when undesired details are near a similar large homogeneous region, in terms of introducing more flattened result. LWTV largely overcomes this shortcoming by adding Log-transform. Particularly, in the first line of Fig. 3, we can observe that RTV cannot discard the white curves near the eyes in the cartoon image. In the second line, the result of RTV destroys the vertical shade shapes on the lower middle of the image. In the third line, RTV severely removes the eyes and nose features on the upper middle of the image. As indicates by black blocks, all of these faults have been largely remedied by our LWTV. Fig. 4 presents the guidance weights of RTV and LWTV, whose intensity values exhibits their differences in describing edges and textures. Specifically, as can be seen in the third column of Fig. 4, the weight values of the background region containing abundant irregular details

${ }^{1}$ For the convenience of reproducible research, matlab code of the LWTV method is available at: https://drive.google.com/drive/mydrive?tmpl=drive. obtained by RTV is much smaller than those of our method. On the other hand, our method endows smaller values for the low contrast edge border. Our adaptive weighting scheme guides the total variation penalty to produce better visual pleasure images.

In Fig. 5, the smoothing results on the Gypsy girl mosaic image are presented. As can be observed, $\mathrm{BF}$ and the model of Buades et al. [13] cannot suppress the texture well, on the other hand, RTV captures the fine details and texture components relatively well, while it overly smooth the slender texture-hair on the left region and faint information on the cheek. The nonlocal scheme Regcovsmooth and our LWTV alleviate these drawbacks. Typically, LWTV exhibits little blurs than those of Regcovsmooth.

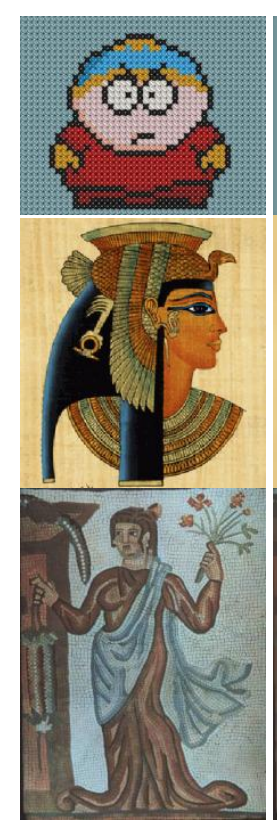

(a)

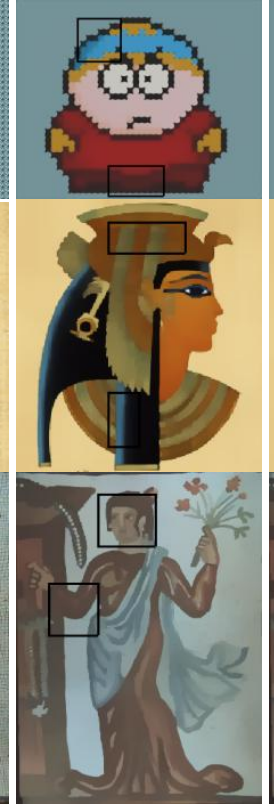

(b)

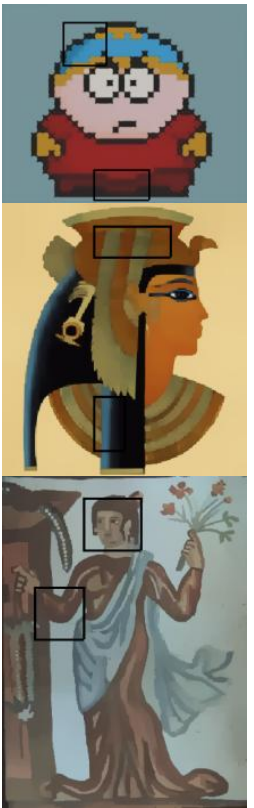

(c)
Fig 3: Image smoothing examples. (a) input, (b) RTV and (c) LWTV. (We suggest readers to take a close look at the results in a high resolution display.)

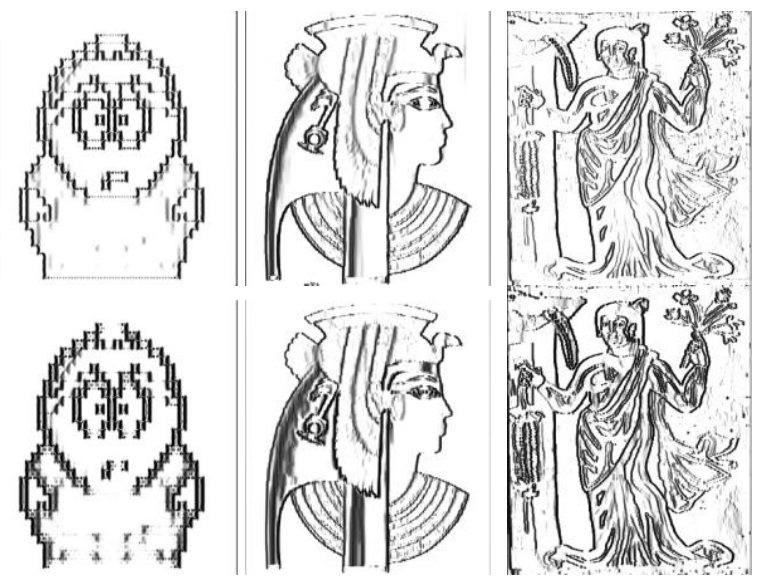

Fig 4: Weight demonstration. Upper: the inversion of normalized $L_{x}$ in RTV, Bottom: $L W_{x}$ value in LWTV. 
In Fig. 6, we provide the smoothing results of the mosaic introduced in Fig. 2. Besides of using different penalty term, both WLS and LWTV use Log-transform as an ingredient of the data-fidelity weight. Their main difference is that one is related to the absolute gradient value of Log-transform at each pixel, and the other is related to the weighted-average signed gradient value of Log-transform in a local window. Their difference can be obviously observed in Fig. 6(a)(c), where LWTV poses better structure extraction from texture simultaneously with edge-preserving capabilities than that of WLS. The result of recent Tree filtering (TF) method with iterative mechanism shown in Fig. 6(b) also indicates that it works inferior to preserve edge than our LWTV filter.

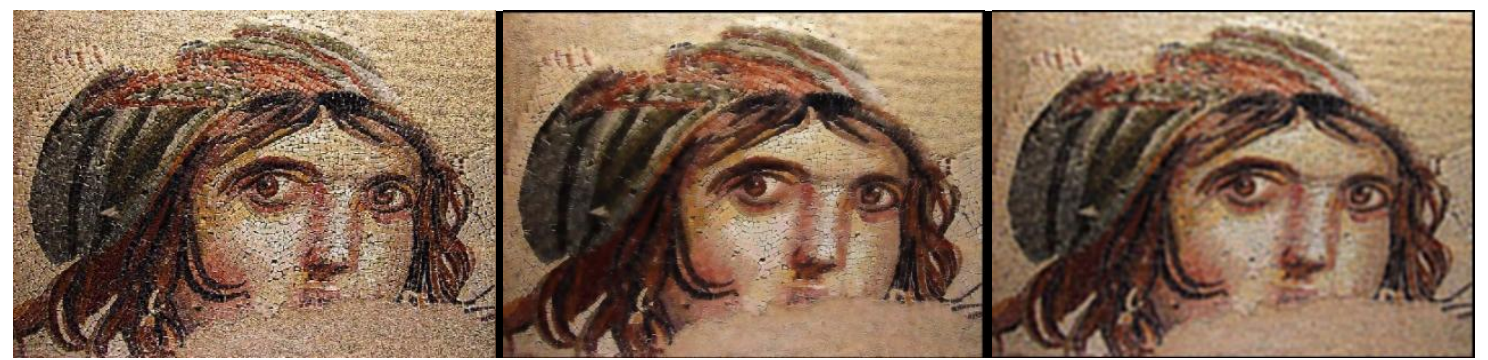

(a) Input image

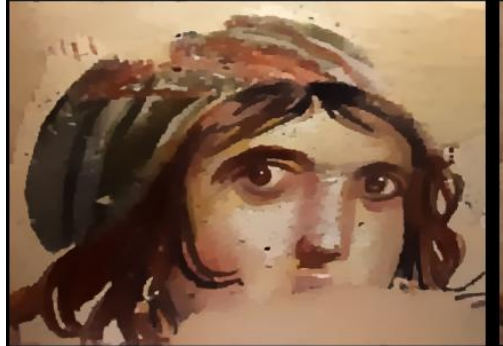

(d) $\operatorname{RTV}(\lambda=0.02, \sigma=3)$ (b) $\operatorname{BF}\left(\sigma_{s}=3, \sigma_{r}=0.3\right)$

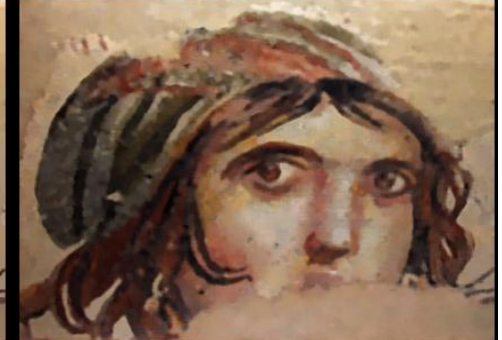

(e) $[5](\sigma=0.2, k=9)$ (c) $[13](\sigma=3.5)$

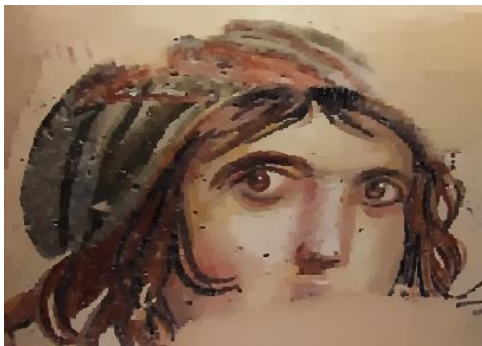

(f) $\operatorname{LWTV}(\lambda=0.1, \sigma=3)$

Fig 5: Smoothing results on the Gypsy girl mosaic image.

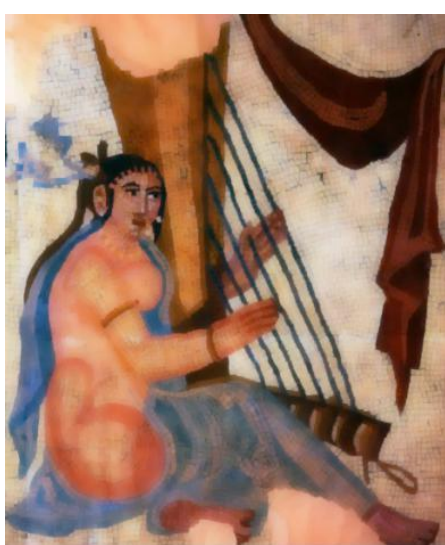

(a) WLS

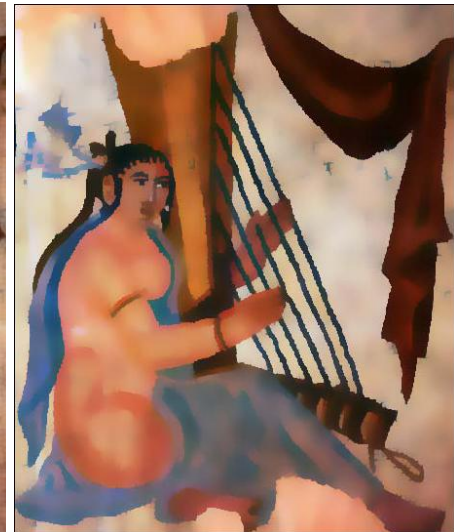

(b) Iterative TF

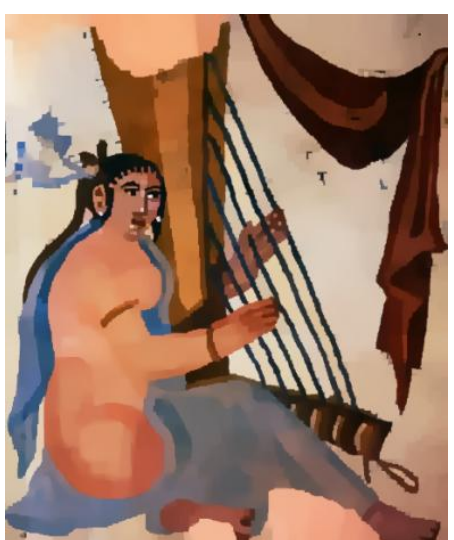

(c) LWTV

Fig 6: Smoothing results on the marble mosaic.

\section{CONCLUSIONS}

This work has presented a new Log-transform signed gradient weighted total variation for smoothing high-contrast details. The decomposition results were substantially improved by forcing larger total variation penalty to the pixel with smaller Log-transform signed gradient weight-average value around its local window. The experimental results demonstrate the effectiveness and robustness of the proposed algorithm, in terms of visual concepts. Further study will investigate this Log-transform based weight in other regularization terms.

\section{ACKNOWLEDGMENTS}

This work was supported in part by the National Natural Science Foundation of China (61261010, 61362001, 62162084, 51165033), the Natural Science Foundation of
Jiangxi Province (20151BAB207008), Jiangxi Advanced Projects for Post-doctoral Research Funds (2014KY02), and Young scientists training plan of Jiangxi province (20133ACB21007, 20142BCB23001).

\section{REFERENCES}

[1] L. Xu, Q. Yan, Y. Xia, J. Jia, "Structure extraction from texture via relative total variation," ACM Trans. Graph., vol. 31, no. 6, 2012.

[2] L. Bao, Y. Song, Q. Yang, H. Yuan, G. Wang, "Tree filtering: efficient structure-preserving smoothing with a minimum spanning tree," IEEE Trans. Image Process., vol. 23, no. 2, pp. 555-569, 2014. 
[3] C. Tomasi, and R. Manduchi, "Bilateral filtering for gray and color images," In ICCV, pp. 839-846. 1998.

[4] Z. Su, X. Luo, Z. Deng, Y. Liang, and Z. Ji, "Edgepreserving texture suppression filter based on joint filtering schemes," IEEE Trans. Multimedia, vol. 15, no. 3, pp. 535-548, Apr. 2013.

[5] L. Karacan, E. Erdem and A. Erdem, "Structure preserving image smoothing via region covariances," ACM Trans. Graph., vol. 32, no. 6, Nov. 2013.

[6] L. Rudin, S. Osher, E. Fatemi, "Nonlinear total variation based noise removal algorithms," Physica D., vol. 60, pp. 259-68, 1992.

[7] Z.Farbman, R. Fattal, D. Lischinski, and R. Szeliski, "Edge-preserving decompositions for multi-scale tone and detail manipulation," ACM Trans. Graph., vol. 27, no. 3, 2008 .

[8] L. Xu, C. Lu, Y. Xu, J. Jia, "Image smoothing via L0 gradient minimization," ACM Trans Graph., vol. 30, no. $6,2011$.
[9] R. C. Gonzalez, R. E. Woods, Digital Image Processing. 3rd ed., Prentice-Hall, Inc., Upper Saddle River, NJ, USA, 2006.

[10] R. Fattal, D. Lischinski, and M. Werman, "Gradient domain high dynamic range compression," In Proc ACM SIGGRAPH, ACM, New York, NY, USA, pp. 249-256. 2002

[11] P. Li and Q. Wang, "Local log-euclidean covariance matrix (12ecm) for image representation and its applications," In Proc. ECCV, vol. 7574, pp. 469-482, 2012.

[12] P. Rodrguez, B. Wohlberg, "Efficient minimization method for a generalized total variation functional," IEEE Trans. Image Process., vol. 18, no. 2, pp. 322-332. 2009.

[13] A. Buades, T. M. Le, J.-M. Morel, and L. A. Vese, "Fast cartoon+texture image filters," IEEE Trans. Image Process., vol. 19, no. 8, pp. 1978-1986, 2010. 